\title{
Advanced systems for public transport scheduling and network design
}

\author{
Hong K. Lo • William H.K. Lam • S.C. Wong • \\ Janny M.Y. Leung
}

Published online: 13 October 2011

(C) Springer-Verlag 2011

This is the third Special Issue related to papers presented at the 11th International Conference on Advanced Systems for Public Transport (CASPT) held in Hong Kong on 20-22 July 2009. First held in Chicago in 1975, the now triennial CASPT conference series serves as a forum for the international community of researchers and practitioners to address all aspects of public transport operations and systems planning.

Faced with the challenges of economic globalization and environmental sustainability, the need for effective and low-energy transport is more urgent than ever. The papers in this Special Issue cover significant contributions in advancing both theory and modeling for optimizing public transit operations and systems design.

The paper by Caimi, Fuchsberger, Laumanns and Schüpbach presents a hierarchical framework for railway timetable design. A schedule on a macroscopic level is first constructed representing desired routes, service frequencies and connections. Next micro-scheduling is done on a regional basis accounting for track allocations

H.K. Lo (凶)

Department of Civil and Environmental Engineering, The Hong Kong University of Science and Technology, Hong Kong, Hong Kong

e-mail: cehklo@ust.hk

W.H.K. Lam

Department of Civil and Structural Engineering, The Hong Kong Polytechnic University, Hong

Kong, Hong Kong

S.C. Wong

Department of Civil Engineering, The University of Hong Kong, Hong Kong, Hong Kong

J.M.Y. Leung

Department of Systems Engineering and Engineering Management, The Chinese University of Hong

Kong, Hong Kong, Hong Kong 
and train speeds. The proposed methodology is applied to the Lucerne region in central Switzerland.

Effective crew scheduling is key to efficient public transit operations. The paper by Nishi, Muroi and Inuiguchi introduces $\underline{\mathrm{dual}}$ valid inequalities within the classical Dantzig-Wolfe column-generation approach to crew-scheduling problems for railways. This is a novel idea and allows the many additional operational constraints (e.g. break scheduling) to be reflected. Computational results indicate that computational times can be significantly reduced.

The paper by Dück, Wesselmann and Suhl also investigates crew scheduling, but in the context of air transport instead of rail. They presented a solution approach that combines column and cut generation. For the pricing sub-problem, they developed a label-setting algorithm with back-tracking. A comprehensive computational study is performed using schedules for a European airline involving hundreds of flights.

The paper by Alt and Weidmann investigates system design issues further by considering the entire public transport chain. They present an overall framework that determines lines/routes and modes (bus, tram, trains) of public transit networks. The optimization is done hierarchically, with the fastest service first, and a genetic algorithm is used to optimize service frequencies and vehicle sizes.

The final paper in this issue, by Li and Quadrifoglio, explores the new and muchdiscussed concept of Demand-Responsive Transit (DRT) for public transport. It explores a model for determining the geographical zones for which DRT should be offered instead of fixed routes, so as to balance customer service quality and operational costs.

This eclectic volume covers a wide range of topics, ranging from new hierarchical approaches for timetabling, methodological advancements for classical crewscheduling problems, to new models and policies for network design for public transit systems. We hope that the papers in this CASPT Special Issue will be of interest to the readership of Public Transport, and also suggest new research questions in systems planning for public transit. 